\title{
When you have eliminated the impossible ...
}

\author{
The Pain Detective: Every Ache Tells a \\ Story: Understanding How Stress and \\ Emotional Hurt Become Chronic Physical \\ Pain \\ Hillel M. Finestone \\ Praeger Publishers; 2009
}

$\mathrm{P}$ ain remains widespread, undertreated and feared by many. Despite growing understanding of its physiology, clinicians have been unable to make substantial progress in treating it effectively. It is estimated that chronic pain affects more than 70 million Americans today and $44 \%$ of the population experiences pain on a regular basis. ${ }^{1}$ The concept of pain has evolved over the years. The contemporary model views it as an experience that is generated in different parts of the brain, and modulated by our emotions, thoughts and memory. The Pain Detective by Dr. Hillel Finestone, offers a fascinating look at the intricate interplay of mind and body, and underscores the importance of emotional and social underpinnings of chronic pain.

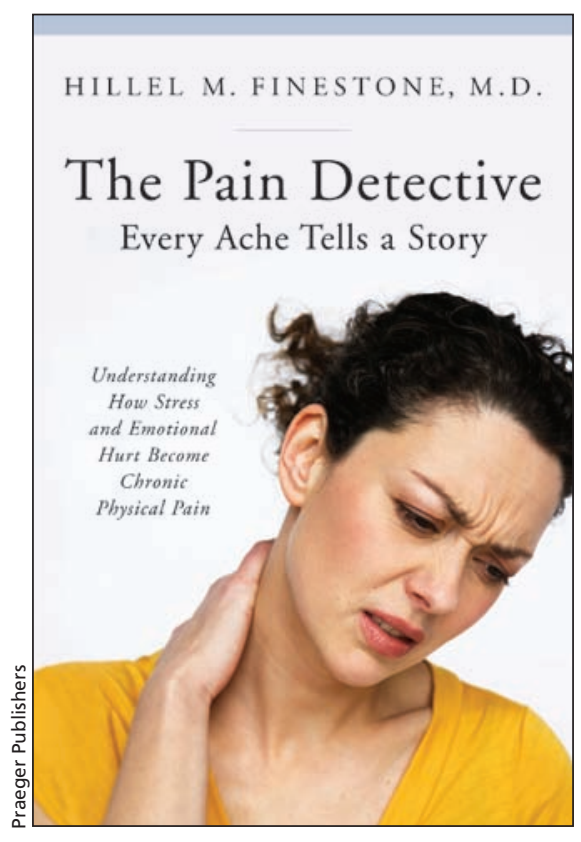

that allow him to trace origins of his patients' pain. The clues discovered during such a process offer a clinician a possible path through which the doctor is able to provide help to patients with pain. As he says, "I am trying to expose

\section{"Pain doesn't kill; it maims. Feelings seem to ignite it, and it seems to ignite feelings."}

Finestone is a physiatrist, a physician who specializes in the field of physical medicine and rehabilitation, and who describes himself as "the pain detective." $\mathrm{He}$ is an astute clinician, but his primary medium is the patients' stories. In the multilayered tapestry of one's emotional life, relationships and challenges of work, he uncovers details the festering wound of emotional and physical hurt. Pain doesn't kill; it maims. Feelings seem to ignite it, and it seems to ignite feelings."

The book is organized around the main themes of musculoskeletal pain: back pain, fibromyalgia and pain in extremities. He also tackles subjects such as chronic fatigue and talks extensively about risk factors for chronic pain: physical and alcohol abuse, and post-traumatic stress disorder. Finestone's tone is conversational and the narration of his cases is accompanied by his commentary. The reader is able to follow the author's thought process, something that I have enjoyed greatly as a pain clinician, and believe many of my colleagues would too. I believe those working in a primary care office will also appreciate the insights gleaned from "being in the room with Dr. F." The purpose of the book, however, is not to learn the elements of physical diagnosis or interviewing skills; rather, it lies in showing the intricate nature of the experience of pain and puts emphasis on the need for treatment that takes that into account.

One of the limitations of the book is that it focuses on one type of pain (i.e., musculoskeletal pain). It does not address the issues of interventional approaches to treatment and it does not mention pharmacologic management. But its strength clearly lies in the stories shared. As Finestone states, "many readers may see pieces of themselves or others somewhere within a particular story and thus be able to help devise recovery plans for themselves, patients, friends, or clients."

\section{Marcin Chwistek MD}

Department of Medical Oncology

Fox Chase Cancer Center

Pain and Palliative Care Program

Philadelphia, $\mathrm{Pa}$.

\section{Reference}

1. Elliott AM, Smith BH, Penny KI, et al. The epidemiology of chronic pain in the community. Lancet 1999;354:1248-52.

CMAJ 2012. DOI:10.1503/cmaj.111452 